\title{
Phase locked loop-based clock synthesizer for reconfigurable analog- to-digital converters
}

\author{
Mateus B. Castro ${ }^{1}$ (D) Raphael R. N. Souza $^{1}$ (D) Agord M. P. Junior $^{1}$ (D) $\cdot$ Eduardo R. Lima $^{2} \cdot$ \\ Leandro T. Manera ${ }^{1}$ (iD
}

Received: 16 March 2021 / Revised: 30 July 2021 / Accepted: 2 August 2021 / Published online: 25 August 2021

(C) The Author(s) 2021

\begin{abstract}
This paper presents the complete design of a phase locked loop-based clock synthesizer for reconfigurable analog-to-digital converters. The synthesizer was implemented in TSMC $65 \mathrm{~nm}$ CMOS process technology and the presented results were obtained from extracted layout view with parasitics. The synthesizer generates clock frequencies ranging from 40 to 230 $\mathrm{MHz}$ considering a reference frequency of $10 \mathrm{MHz}$ and a supply voltage of $1.2 \mathrm{~V}$. Worst case current consumption is 634 $\mu \mathrm{W}$, settling time is $6 \mu \mathrm{s}$, maximum jitter is $1.3 \mathrm{~ns}$ in a $0.037 \mathrm{~mm}^{2}$ area. Performance was validated in a test $\Sigma \Delta \mathrm{Modulator}$ with bandwidths of $200 \mathrm{kHz}, 500 \mathrm{kHz}$ and $2 \mathrm{MHz}$, and oversampling frequencies of 40, 60 and $80 \mathrm{MHz}$ respectively, with negligible signal-to-noise ratio degradation compared to an ideal clock.
\end{abstract}

Keywords PLL $\cdot$ Clock $\cdot$ ADC $\cdot$ Wireless

\section{Introduction}

With the rising number of wireless standards in the past decades either with the evolution and wide adoption of Bluetooth and Global Positioning System (GPS) in mobile devices, the continuous use of $3 \mathrm{G}$ technology, popularization of $4 \mathrm{G}$ technologies and the recent introduction of $5 \mathrm{G}$ in commercial products, receiver reconfigurability becomes an interesting and beneficial feature in Systemon-Chip (SoC) embedded devices that require a large

Mateus B. Castro

mateus.castro@ieee.org

Raphael R. N. Souza

raphael.souza@ieee.org

Agord M. P. Junior

agord@dsif.fee.unicamp.br

Eduardo R. Lima

eduardo.lima@eldorado.org.br

Leandro T. Manera

leandro@dsif.fee.unicamp.br

1 University of Campinas, Campinas, SP, Brazil

2 Eldorado Research Institute, Campinas, SP, Brazil variety of Radio Frequency (RF) standards for signal processing.

One possible way of reconfiguration in wireless receivers (depicted in Fig. 1) is in the Analog-to-Digital Converter (ADC). By varying its clock sampling frequency, the output data rate can be reduced for a particular standard and thus allowing for lower power consumption when fast conversion is not necessary.

In this paper, an integer-N Phase Locked Loop-based (PLL) clock synthesizer is designed to provide the necessary frequencies for two different ADC architectures. The first ADC architecture is based on a Sigma-Delta structure for GSM, Bluetooth and UMTS standards, and the second architecture is based on Pipelined structure, employed for DVB-S2 standard.

Signal-to-Noise ratio (SNR) degradation due to clock jitter is a major problem and becomes more significant in higher frequencies regardless of ADC topology [1]. Another point of concern in large SoCs is the area availability for each sub-circuit. This was a major constraint during the design phase of this project, limiting the possible topologies of voltage controlled oscillators (VCOs).

This paper is organized as follows: Sect. 2 presents a general overview of the proposed PLL, followed by the circuit design of the main sub-blocks of the system in Sect. 


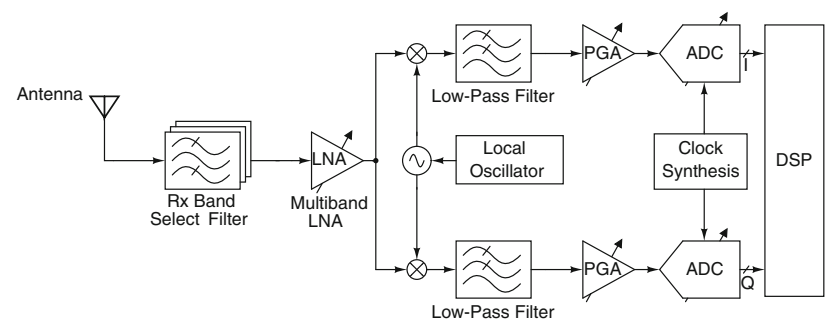

Fig. 1 Reconfigurable direct conversion receiver

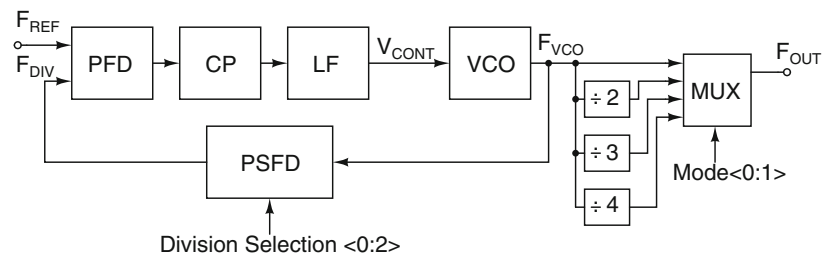

Fig. 2 PLL architecture block diagram
Table 2 PLL Frequency planning

\begin{tabular}{llcll}
\hline Mode & DVB-S2 & UMTS & BT & GSM \\
\hline F $_{\text {MIN }}(\mathrm{MHz})$ & 160 & 80 & 60 & 40 \\
F $_{\text {MAX }}(\mathrm{MHz})$ & 230 & 135 & 76.66 & 67.5 \\
\hline
\end{tabular}

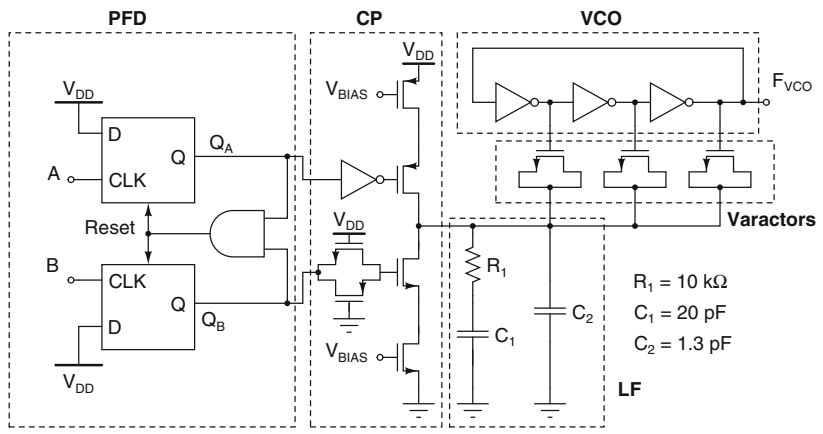

Fig. 3 PLL feedforward path: PFD, CP, LPF and VCO
Table 1 PLL frequency requirements

\begin{tabular}{lllll}
\hline Standard & ADC type & Sampling freq. & Bandwidth & Max. Jitter \\
\hline GSM & $\Sigma \Delta$ & $40 \mathrm{MHz}$ & $200 \mathrm{kHz}$ & $\leq 10 \mathrm{~ns}$ \\
Bluetooth & $\Sigma \Delta$ & $60 \mathrm{MHz}$ & $500 \mathrm{kHz}$ & $\leq 5 \mathrm{~ns}$ \\
UMTS & $\Sigma \Delta$ & $2 \mathrm{MHz}$ & $80 \mathrm{MHz}$ & $\leq 3 \mathrm{~ns}$ \\
DVB-S2 Mode 1 & Pipeline & 80 to $120 \mathrm{MHz}$ & 80 to $120 \mathrm{MHz}$ & $\leq 50 \mathrm{ps}$ \\
DVB-S2 Mode 2 & Pipeline & 160 to $200 \mathrm{MHz}$ & 160 to $200 \mathrm{MHz}$ & $\leq 30 \mathrm{ps}$ \\
\hline
\end{tabular}

3. In Sect. 4 the results are presented followed by the conclusion in Sect. 5 .

\section{PLL based clock synthesizer overview}

The complete diagram of the proposed PLL is shown in Fig. 2 and its operation is as follows: first $F_{R E F}$ of $10 \mathrm{MHz}$ is compared with the feedback $F_{D I V}$ frequency, which is the voltage-controlled oscillator (VCO) output frequency $F_{V C O}$ divided by the pulse-swallow frequency divider (PSFD). The comparison result is fed to the charge pump (CP) circuit, which injects a current in the loop filter (LF), altering the control voltage $V_{C O N T}$. This feedback scheme controls the VCO output frequency, until the system reaches locked condition in the steady-state operation. The output VCO frequency can also be divided by two, three or four, resulting in $F_{\text {OUT }}$.

The clock frequency requirements obtained from the ADCs specifications are presented in Table 1 and the frequency planning is shown in Table 2. Jitter requirements of Table 1 are taken from [2], based on (1)
$S N R=-20 \log \left(2 \pi f_{i n} \sigma\right)$

where SNR is the ADCs Signal-to-Noise Ratio, $f_{\text {in }}$ is the maximum input frequency and $\sigma$ is the rms value of jitter.

\section{Circuit design}

\subsection{PFD, CP, LPF and VCO}

The designs of the phase and frequency detector (PFD), CP, LF and VCO are shown in Fig. 3.

The first sub-circuit of the PLL system is the PFD. As the name implies, it detects the phase an frequency from two waveforms A and B shown in Fig. 3. It is a simple and robust design consisting of two resettable D flip-flops controlled by an AND gate [3].

After the phase and frequency detection performed by the PFD, the signals $Q_{A}$ and $Q_{B}$ control if the CP sinks our sources charge in the LF. An always-on transmission gate was used at the input $Q_{B}$ to avoid skewing due to the delay introduced by the inverter at the $Q_{A}$ input and therefore 


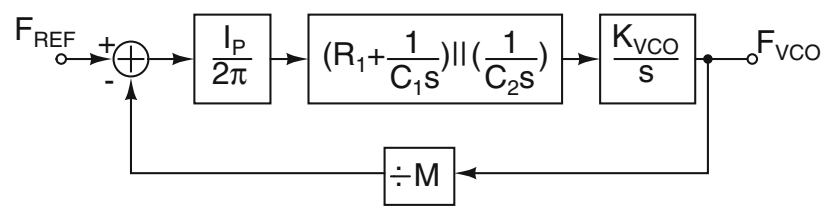

Fig. 4 PLL linear model: frequency domain representation

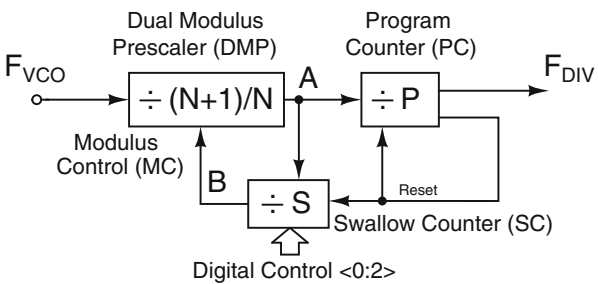

Fig. 5 Pulse swallow frequency divider (PSFD) architecture

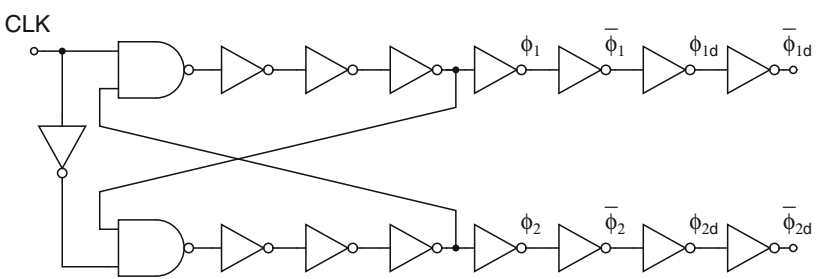

Fig. 7 Non-overlapping clock generation

closed loop transfer function settling behavior is negligibly affected [3]. Based on the linear model of Fig. 4, its transfer function can be written as (2) [4]

$$
H(s)=K^{\prime} \frac{s+1 /\left(R_{1} C_{1}\right)}{s^{3}+\frac{1}{R \frac{C_{1} C_{2}}{C_{1}+C_{2}}} s^{2}+K^{\prime} s+K^{\prime} \frac{1}{R_{1} C_{1}}},
$$

Program Counter (PC)

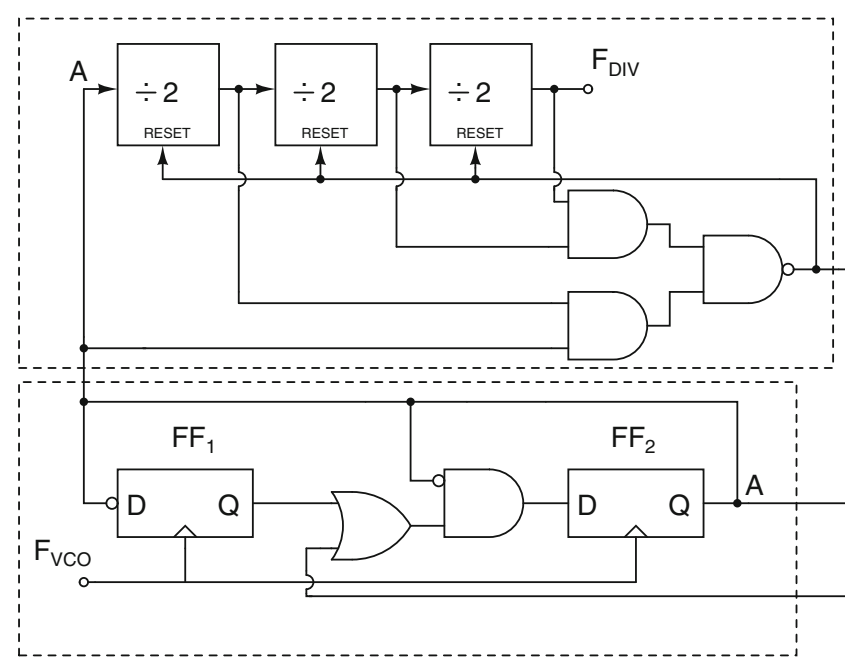

Dual Modulus Prescaler (DMP)

Fig. 6 Complete design of the pulse swallow divider

equalizing the delays in both control switches. To avoid the imbalance of the up and down currents wider transistors were chosen for the current sources. The increased capacitance from larger transistor did not impact the PLL operation as the synthesized frequencies are not very high.

The basic idea of the capacitor $C_{1}$ is to store or release the charge coming from the $\mathrm{CP}$, and control the voltage $V_{C O N T}$. The VCO adds another pole at the origin, thus, through the addition of resistor $R_{1}$ in series with $C_{1}$, the loop is stabilized with the addition of a zero at $-1 / R_{1} C_{1}$. The addition of $R_{1}$ introduces a ripple in the $V_{C O N T}$ waveform, and hence spurs in the spectrum. The magnitude of the ripple is proportional to the resistor size and the current coming from the $\mathrm{CP}$. To reduce the ripple, the capacitor $C_{2}$ is introduced. Choosing $C_{2} \leq 0.2 C_{1}$, the where $K_{V C O}$ is the VCO gain, $I_{p}$ is the $\mathrm{CP}$ current, $M$ is the division ratio of the PLL and $K^{\prime}$ is

$K^{\prime}=\frac{K_{V C O} I_{P}}{2 \pi M C_{2}}$.

One of the requirements during development of the DVBS2 SoC project was a small area availability for the PLL clock synthesizer. Thus an optimum choice of VCO topology is the inverter-based voltage-controlled ring oscillator (VCRO) as it will combine small area and low power consumption. Three inverters are connected in series, with the output of the third inverter connected to the input of the first. The oscillation frequency control is made by the source-gate-bulk voltage in the n-type MOSFET varactors connected to each inverter output. The varactors 


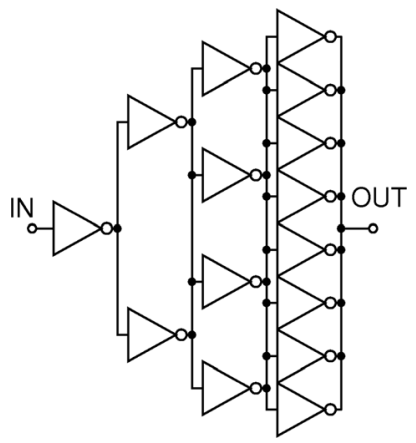

Fig. 8 Buffer tree for each clock phase

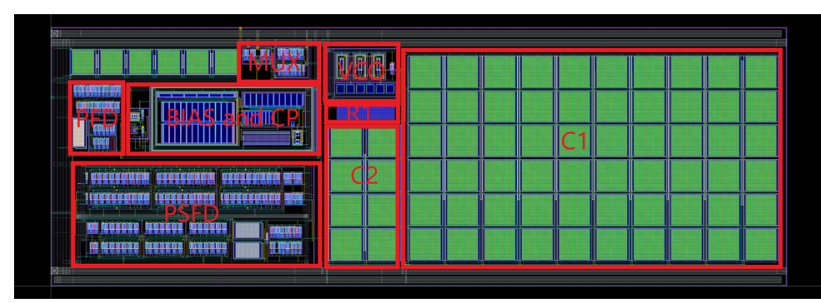

Fig. 9 Implemented PLL layout view

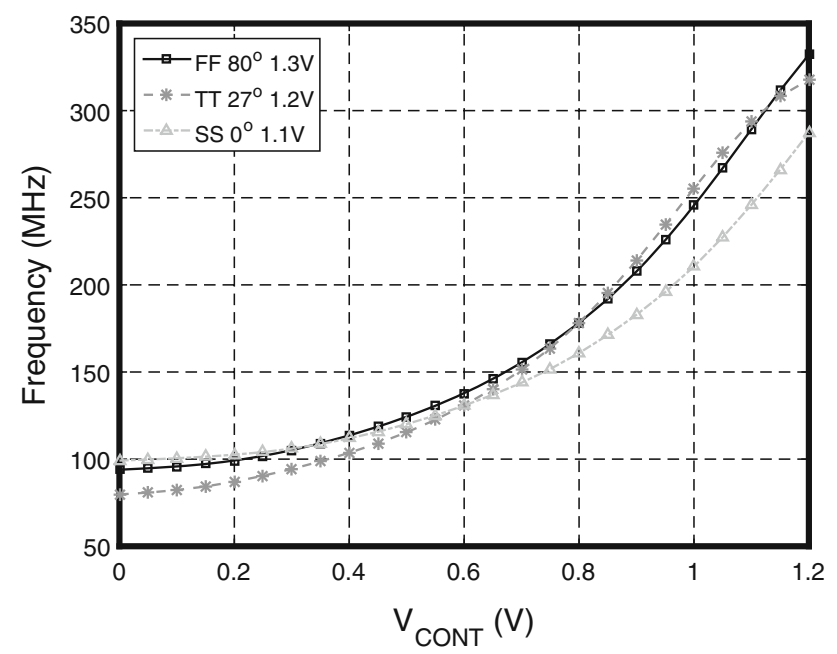

Fig. 10 VCO frequency tuning characteristic

decrease in capacitance as $V_{C O N T}$ (Fig. 3) increases, hence the oscillation frequency increases.

\subsection{Programmable loop frequency divider}

From the specifications defined by Table 1 , an integer-N second order PLL-based frequency synthesizer is a proper structure to obtain all desired frequencies. Therefore, the Pulse-Swallow Frequency Divider (PSFD) was chosen. The overall divide ratio $\left(M=F_{V C O} / F_{R E F}\right.$ in Fig. 2$)$ can be adjusted only by a programmable counter named Swallow Counter (SC) in Fig. 5.

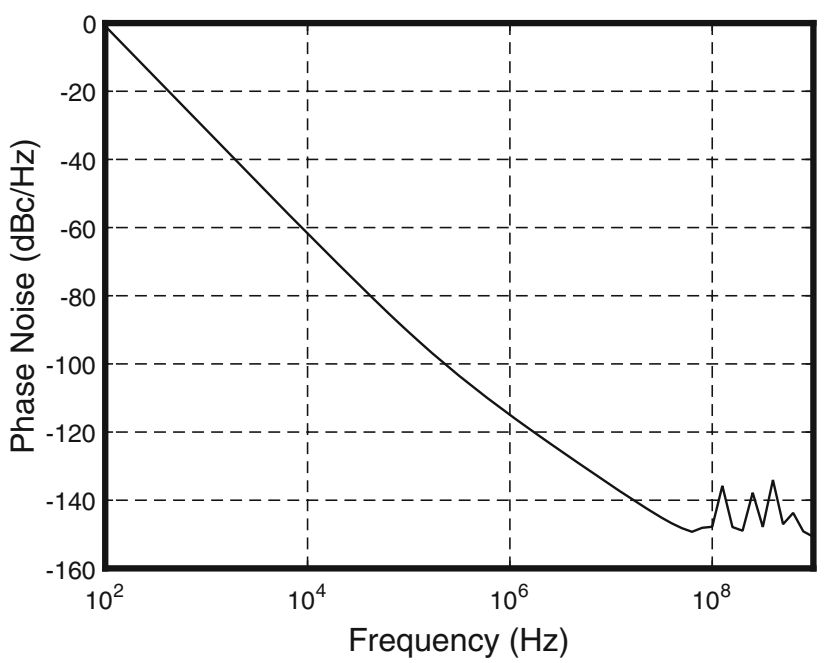

Fig. 11 VCO phase noise in worst case (FF, $\left.1.3 \mathrm{~V}, 80^{\circ} \mathrm{C}\right)$

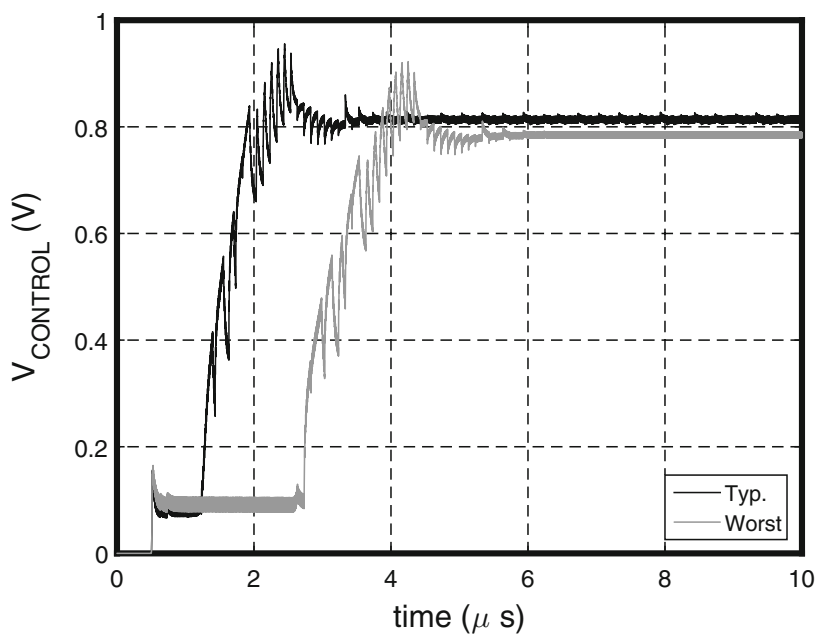

Fig. 12 Control voltage comparison in typical conditions (TT, $1.2 \mathrm{~V}$ $27^{\circ} \mathrm{C}$ ) and worst case (SS, $1.1 \mathrm{~V}, 0^{\circ} \mathrm{C}$ ) for $200 \mathrm{MHz}$

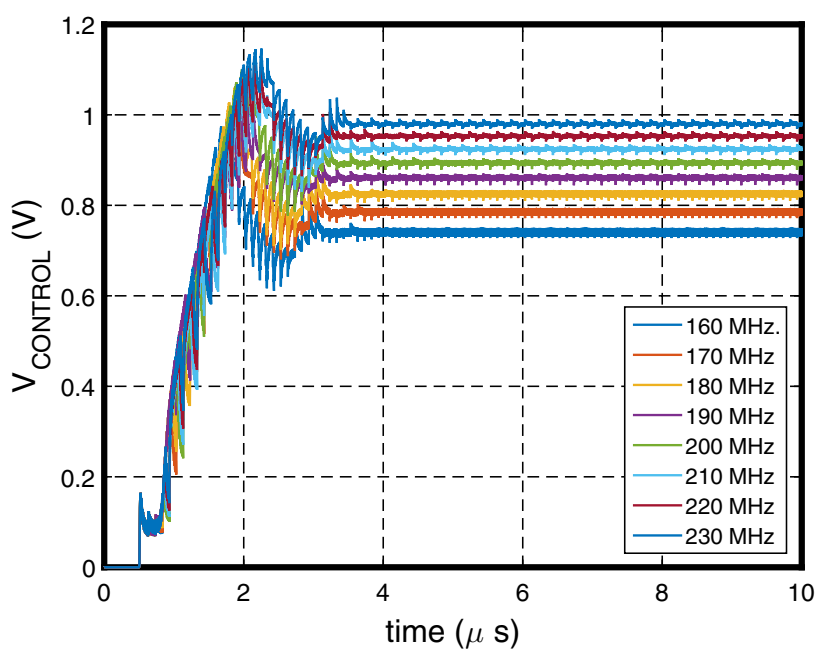

Fig. 13 Control voltage comparison for all frequency selections, varying from 160 to $230 \mathrm{MHz}$ 


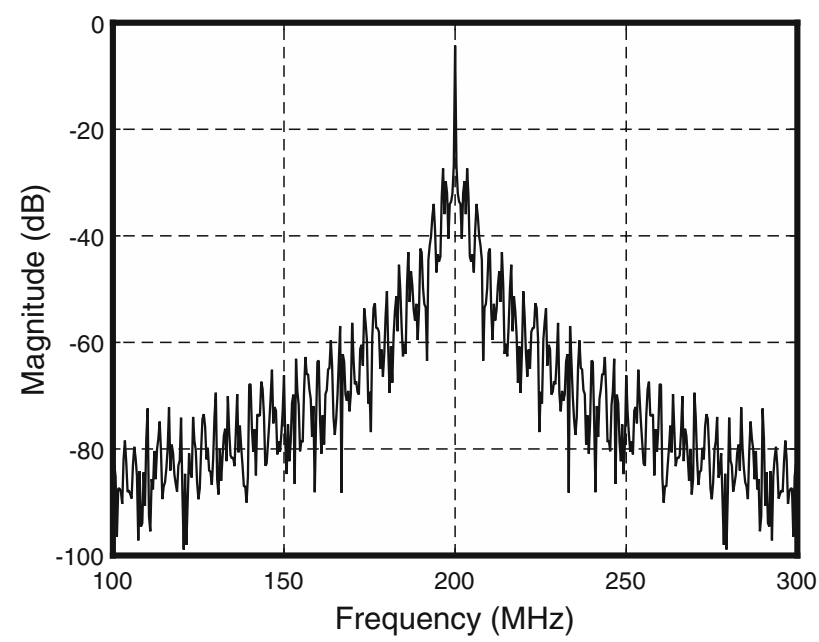

(a) Typical conditions (TT, $1.2 \mathrm{~V}, 27^{\circ} \mathrm{C}$ ) for $200 \mathrm{MHz}$

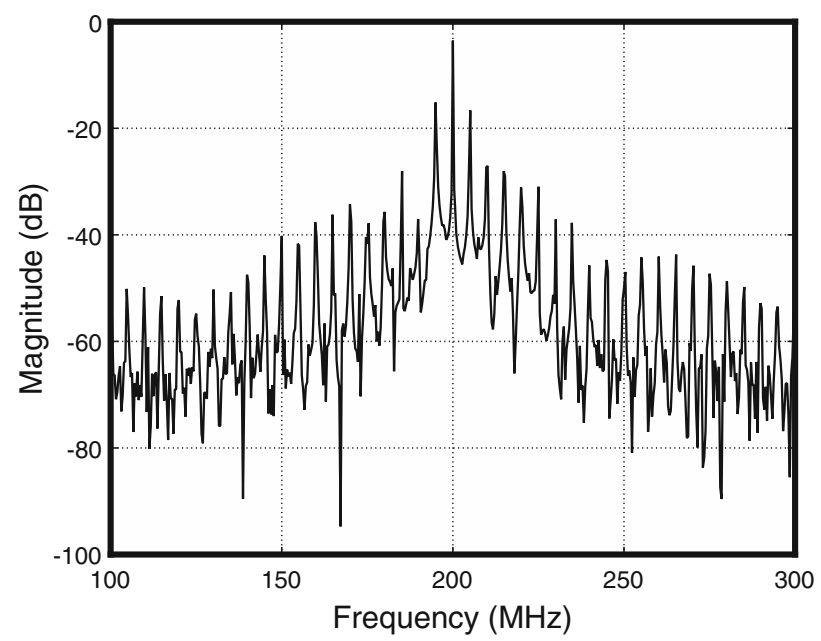

(b) Worst case conditions ( $\mathrm{FF}, 1.3 \mathrm{~V}, 80^{\circ} \mathrm{C}$ ) for $200 \mathrm{MHz}$

Fig. 14 Spectra for typical (a) and worst case (b) when $200 \mathrm{MHz}$ is selected

From Fig. 2, the operation of the PSFD can be described as follows: initially $\mathrm{P}$ and $\mathrm{S}$ are reset and the frequency at A is equal to $F_{V C O} /(N+1)$. After $(N+1) S$ pulses, the $\mathrm{SC}$ changes $\mathrm{B}$ to HIGH. Now the frequency at $\mathrm{A}$ is equal to $F_{V C O} / N$. After $(P-S)$ pulses, $\mathrm{P}$ goes HIGH and resets the $\mathrm{SC}$, restarting the process. The resulting output frequency is $F_{D I V}=F_{V C O} /(N P+S)$ as long as $P>S$.

In this design the values $\mathrm{N}, \mathrm{P}$ and $\mathrm{S}$ are 2,8 and 7 respectively, allocating three bits for the SC control and allowing the reference frequency to be multiplied by 16 up to 23 .

The Dual Modulus Prescaler (DMP) is shown in Fig. 6 and consists of a divide by 3 with a modified logic to accommodate an external selection bit. When Modulus Control (MC) is HIGH, $F F_{1}$ has no effect and the circuit

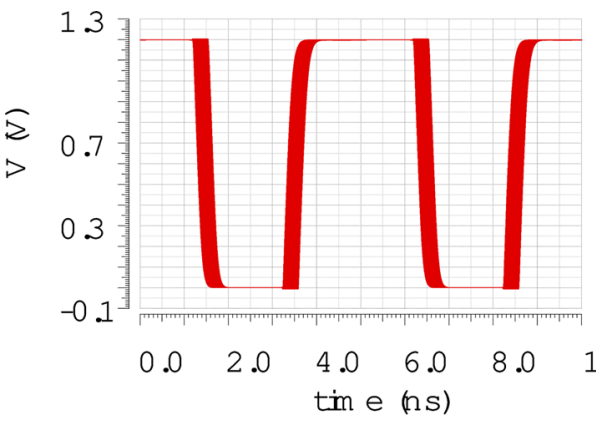

(a) Eye diagram in typical case

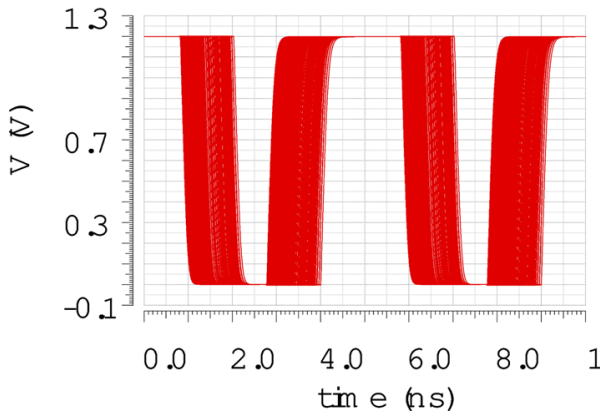

(b) Eye diagram in worst case

Fig. 15 Eye diagram for a typical (TT, $1.2 \mathrm{~V}, 27^{\circ} \mathrm{C}$ ) and $\mathbf{b}$ worst (FF, $1.3 \mathrm{~V}, 80^{\circ} \mathrm{C}$ ) cases

Table 3 PLL performance summary

\begin{tabular}{llll}
\hline Parameter & Min. & Typ. & Max. \\
\hline Supply voltage $(\mathrm{V})$ & 1.1 & 1.2 & 1.3 \\
Power $(\mu \mathrm{W})$ & 299 & 385 & 634 \\
Freq. Range $(\mathrm{MHz})$ & 40 & - & 260 \\
$\mathrm{~K}_{\mathrm{VCO}}(\mathrm{MHz} / \mathrm{V})$ & 156.8 & 198.4 & 198.5 \\
Settling time $^{a}(\mu \mathrm{s})$ & 3.7 & 4 & 6 \\
Maximum overshoot $(\mathrm{V})_{\text {Jitter }^{a}(\mathrm{~ns})}^{0.96}$ & 1.1 & 1.15 \\
Maximum Spur $^{a}(\mathrm{~dB})$ & 0.3 & 0.5 & 1.3 \\
Area $\left(\mu \mathrm{m}\left(\mathrm{mm}^{2}\right)\right)$ & -60 & -29 & -18 \\
\hline
\end{tabular}

${ }^{a}$ Measured when $200 \mathrm{MHz}$ is selected

divides by 2 . If $\mathrm{MC}$ is LOW, the circuit divides by 3 . Fig. 6 also presents the $\mathrm{SC}$ design, it is a slight variation from the design present in [3]. The input frequency is counted from 000 up to the word defined by $\mathrm{D}<0>, \mathrm{D}<1>$ and $\mathrm{D}<2>$. Then, it changes the output to HIGH, altering the DMP division, disabling all dividers while it retains the output on HIGH until the Program Counter (PC) resets all dividers. Now the output returns to LOW and the process is repeated. The PC is a simple divide by 8 circuit with an end of count reset, also shown in Fig. 6. 
Table 4 Comparison with other works

\begin{tabular}{llllll}
\hline & This Work & {$[6]$} & {$[7]$} & {$[8]$} & {$[9]$} \\
\hline Type & PLL & PLL & PLL & DTC-DDS & PLL \\
Design Phase & Layout & Fabricated & Fabricated & Simulation & Layout \\
Ref. Freq. (MHz) & 10 & 20 & 20 & 100 & 6.25 \\
Tunning Range (Hz) & $40-230 \mathrm{M}$ & $200 \mathrm{M}$ & $100-560 \mathrm{M}$ & $4.7-5 \mathrm{G}$ & $100-300 \mathrm{M}$ \\
Tech. (nm) & 65 & 90 & 180 & 65 & 250 \\
Supply $(\mathrm{V})$ & 1.2 & 1.2 & 3.3 & 1.2 & 3.3 \\
Area $\left(\mathrm{mm}^{2}\right)$ & 0.037 & 0.18 & 0.16 & $\mathrm{n} / \mathrm{a}$ & Not informed \\
Power $(\mathrm{mW})$ & 0.634 & 9 & 24 & 20 & 6 \\
Jitter $(\mathrm{ps})$ & 1300 & 3 & 4 & 1.1 & 12 \\
\hline
\end{tabular}

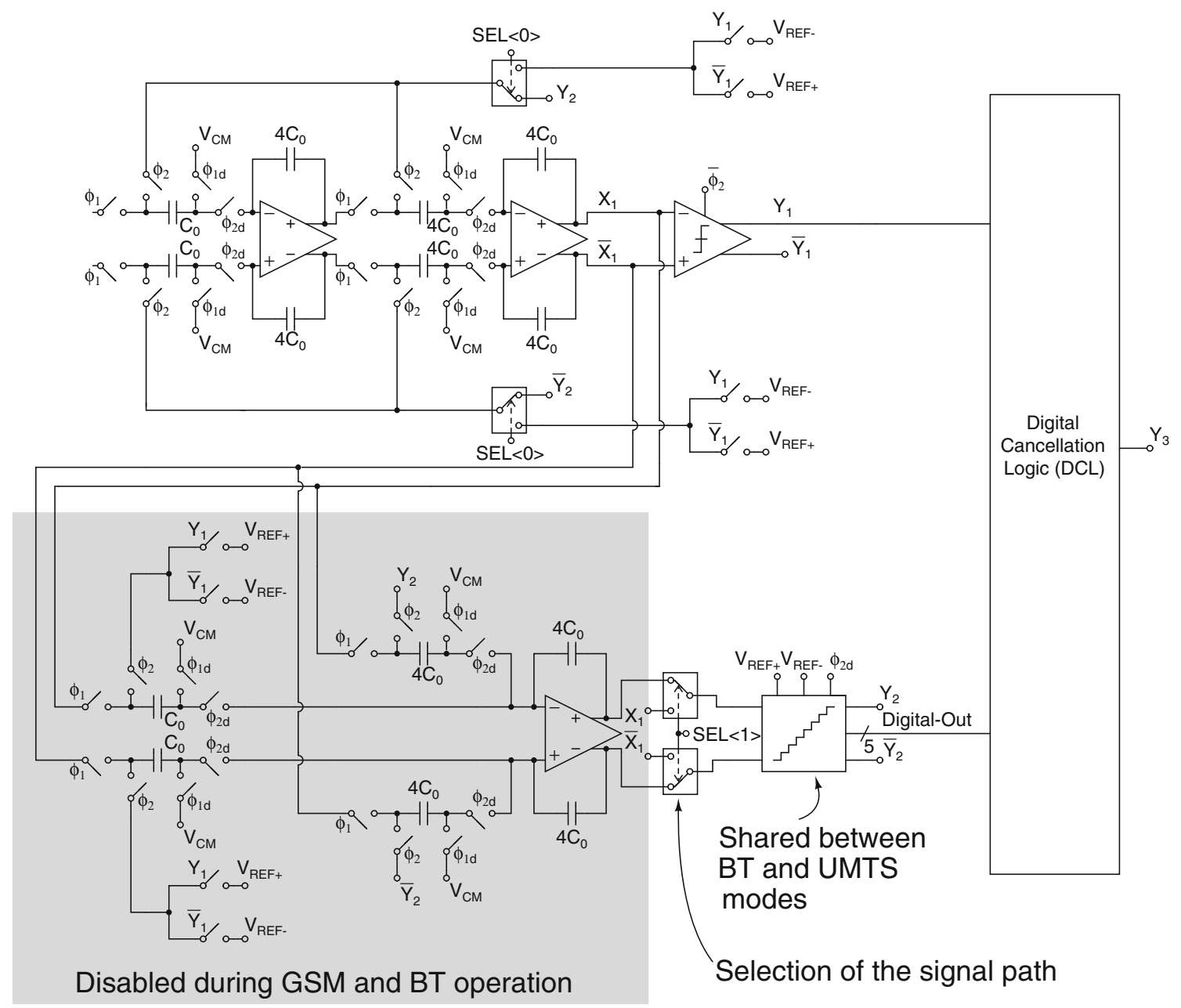

Fig. 16 Reconfigurable $\Sigma \Delta$ Modulator under test

\subsection{Non-overlapping clock generation and clock buffers}

Generally in switched-capacitor circuits two phases of nonoverlapping clocks are required to avoid unwanted charge transfer during sampling and transfer stages. A common design implementation of non-overlapping clock phases $\phi_{1}$ and $\phi_{2}$ is shown in Fig. 7. Additional phases $\phi_{3}$ and $\phi_{4}$ are delayed versions of $\phi_{1}$ and $\phi_{2}$

To drive large capacitors in the ADCs the clock phases require proper buffering. This is achieved by the buffer tree shown in Fig. 8 for each phase [5]. 
Table 5 Comparison between ideal and worst case clock results in an $\Sigma \Delta$ Modulator

\begin{tabular}{|c|c|c|c|c|c|c|}
\hline \multicolumn{2}{|l|}{ Param./Mode } & GSM & \multicolumn{2}{|c|}{ Bluetooth } & \multicolumn{2}{|c|}{ UMTS } \\
\hline \multicolumn{2}{|c|}{ Samp. Freq. (MHz) } & 40 & \multicolumn{2}{|c|}{60} & \multicolumn{2}{|l|}{80} \\
\hline \multicolumn{2}{|c|}{ Bandwidth (MHz) } & 0.2 & \multicolumn{2}{|c|}{0.5} & \multicolumn{2}{|l|}{2} \\
\hline \multicolumn{2}{|c|}{ Modulator Order } & $2^{\text {nd }}$ & \multicolumn{2}{|c|}{$2^{\text {nd }} 2.5$ bit } & \multicolumn{2}{|c|}{$3^{\text {rd }} 2.5 \mathrm{bit}$} \\
\hline Clock Type & Ideal & PLL & Ideal & PLL & Ideal & PLL \\
\hline SNR (bits) & 12.78 & 12.6 & 12 & 11.68 & 8.9 & 9 \\
\hline SFDR (dB) & 84.3 & 82 & 80 & 75.2 & 65.2 & 66.23 \\
\hline
\end{tabular}

\section{Results}

All simulations were performed using Cadence Virtuoso environment in a extracted layout view, including parasitics and corners for process, supply variation and temperature. The PLL design was realized in TSMC $65 \mathrm{~nm}$ process. For the LF, Metal-Oxide-Metal (MoM) capacitors were used, while for the $\Sigma \Delta$ Modulator circuit validation, Metal-Insulator-Metal (MiM) capacitors were used. PolyResistors were used in both designs. Complete layout is presented in Fig. 9. The total area is $114 \times 324 \mu \mathrm{m}$ $\left(0.037 \mathrm{~mm}^{2}\right)$.

The VCO frequency characteristic varying with the control voltage is shown in Fig. 10 and the phase noise for worst case $\left(\mathrm{FF}, 1.3 \mathrm{~V}, 80^{\circ} \mathrm{C}\right)$ is shown in Fig. 11 . The phase noise performance, of around $-90 \mathrm{dBc} / \mathrm{Hz}$ at $100 \mathrm{kHz}$ frequency offset, is the expected for the ring oscillator topology. Improvements in phase noise response can be achieved at the expense of power consumption [3].

Figure 12 presents the settling behavior of the control voltage fed to the VCO when $200 \mathrm{MHz}$ is selected. Worst case condition in this case is specifically for total settling time, which happens with slow-slow devices at $0^{\circ} \mathrm{C}$ and with supply at $1.1 \mathrm{~V}$ resulting in total settling time of about $6 \mu \mathrm{s}$. Although the worst case results in worst settling time, the ripple in settled condition is actually smaller than typical operation, resulting in a cleaner spectrum, and less jitter, which is desirable for the proposed application since there are no strict requirements in regards to settling time. Figure 13 presents the settling behavior for each possible frequency selection defined by the PSFD.

Figure 14(a), (b) show the obtained spectrum for a selected frequency of $200 \mathrm{MHz}$ in typical conditions (TT devices, supply at $1.2 \mathrm{~V}$ and $27^{\circ} \mathrm{C}$ ) and worst case condition (FF devices, supply at $1.3 \mathrm{~V}$ and $80^{\circ} \mathrm{C}$ ) respectively, spanning between $100 \mathrm{MHz}$ and $300 \mathrm{MHz}$. The spectrum showed severe degradation as devices tend to fall in the fast-fast process variation.
Jitter response was obtained by eye diagram plot performed in Cadence Virtuoso environment shown in Fig. 15. As expected from spectrum curves, jitter showed to be worst in fast-fast, $1.3 \mathrm{~V}$ supply voltage and $80^{\circ} \mathrm{C}$ condition, resulting in $1.3 \mathrm{~ns}$, while in typical conditions the resulting jitter is 500 ps. Of course this simulations will only capture the deterministic portion of the total jitter, however this verification is a sufficient preliminary result to give an idea of the PLL behavior in worst case conditions.

Performance summary of the PLL is presented in Table 3 and comparison with other works is shown in Table 4. It is clear that compared with other works, this design trades power dissipation for jitter degradation.

Validation of the generated clock was performed in the reconfigurable $\Sigma \Delta$ Modulator shown in Fig. 16. The nonoverlapping clocks are generated by the circuit of Fig. 7 and are inserted in the switches of modulator highlighted by the symbols $\phi_{1}, \phi_{2}, \phi_{1 d}$ and $\phi_{2 d}$ of Fig. 16. Clock frequencies for each case and results compared with an ideal clock are summarized in Table 5. The tests were performed with clocks at 40,60 and $80 \mathrm{MHz}$, in a second order modulator for 40 and $60 \mathrm{MHz}$ tests and third order for the $80 \mathrm{MHz}$ test.

GSM, Bluetooth and UMTS bandwidths, obtained values of Signal-to-Noise Ratio (SNR) and Spurious Free Dynamic Range (SFDR) are in accordance with several reported reconfigurable $\Sigma \Delta$ modulators such as [10] and [11]. Modulator spectra are presented in Fig. 17 comparing ideal clock and worst case PLL generated clock for each case. Overall the results showed negligible degradation in SNR and no noticeable additional spurs are present in the spectra, confirmed by the obtained values of SFDR. Although the SNR degradation proved more severe in the $60 \mathrm{MHz}$ case, the results are still satisfactory and validate the clock synthesizer design for the $\Sigma \Delta$ type ADC.

From Table 1, jitter performance requirements for the Pipeline topology were not met. Several jitter and duty cycle correction circuits have been reported $[12,13]$ and will be necessary alongside calibration techniques to improve SNR degradation.

\section{Conclusions}

A PLL-based clock generator to be used in reconfigurable ADCs was designed to generate clocks in the span between 40 and $230 \mathrm{MHz}$. Results were validated in extracted layout simulations including parasitics and PVT corners. Additional validation was performed in a prototype reconfigurable $\Sigma \Delta$ Modulator for oversampling frequencies of 40, 60 and $80 \mathrm{MHz}$, with no significant degradation in performance compared with the results of an ideal clock. 


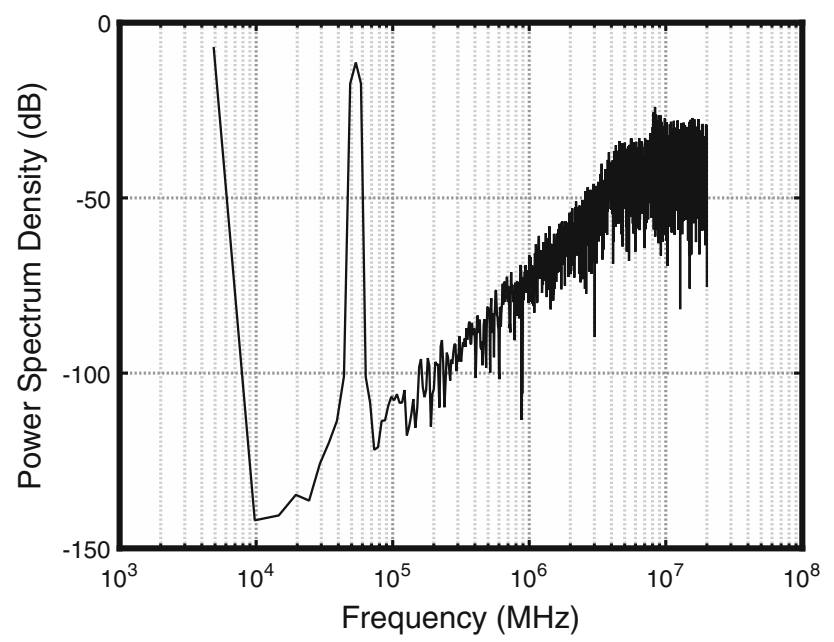

(a) Ideal clock at $40 \mathrm{MHz}$

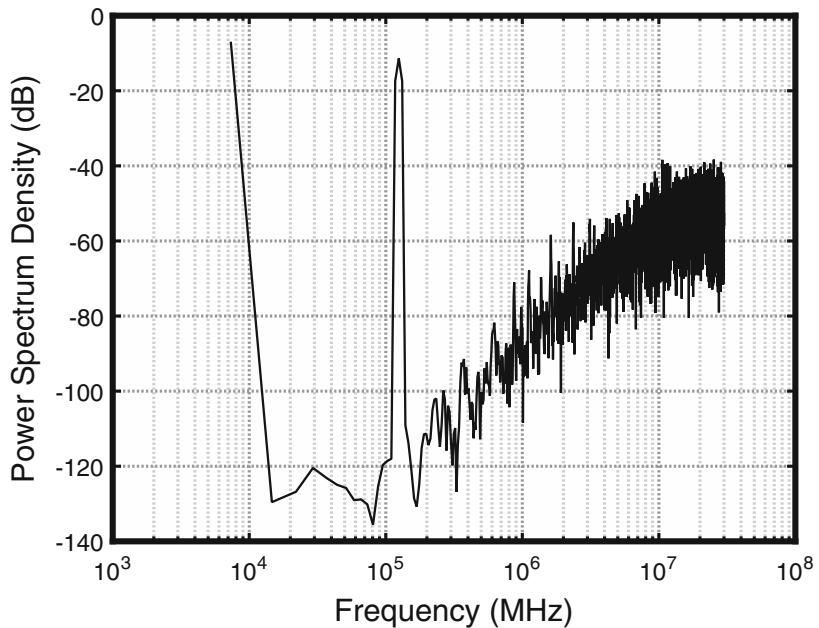

(c) Ideal clock with $60 \mathrm{MHz}$

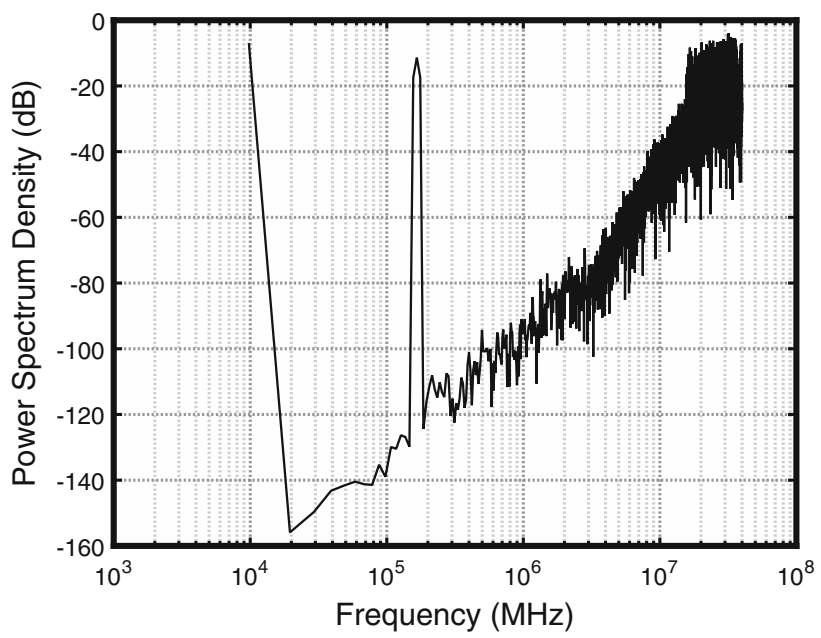

(e) Ideal clock with $80 \mathrm{MHz}$

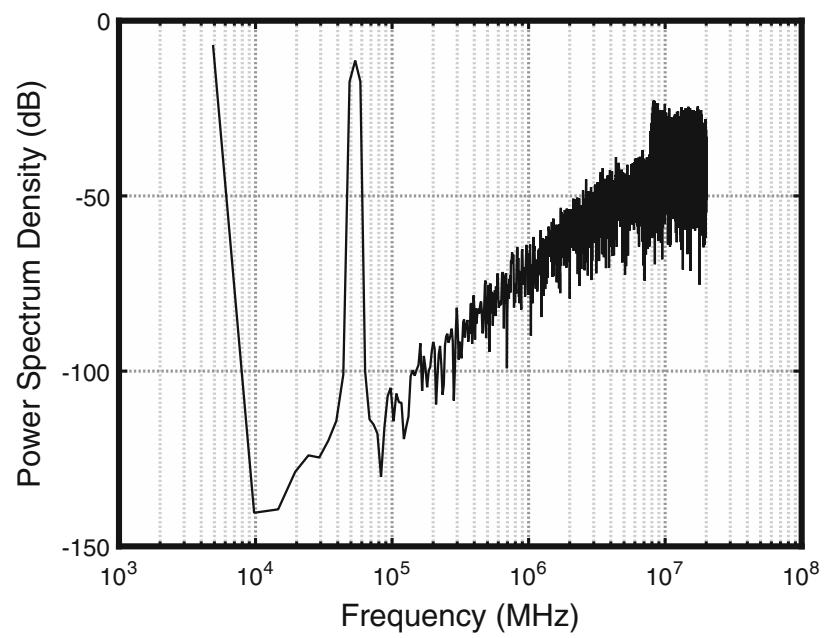

(b) PLL-based clock at $40 \mathrm{MHz}$

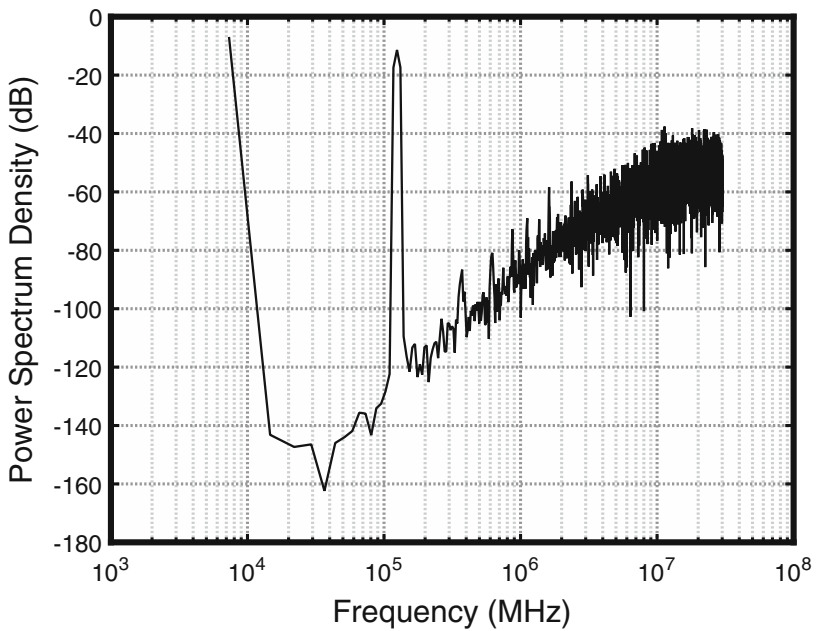

(d) PLL-based clock at $60 \mathrm{MHz}$

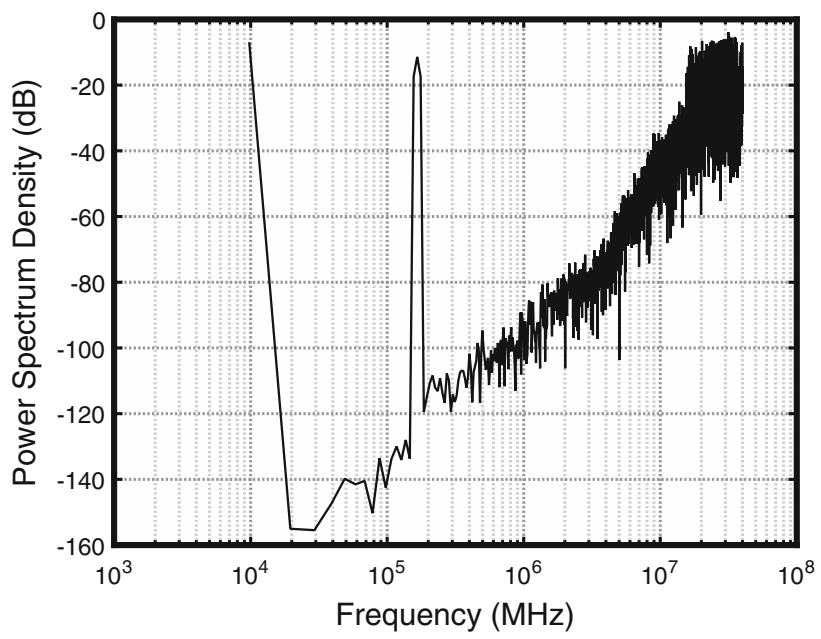

(f) PLL-based clock at $80 \mathrm{MHz}$

Fig. 17 Power Spectrum Density comparison between ideal clock and PLL-based clock for different oversampling frequencies. Hanning Window with $8192 \mathrm{FFT}$ points. $53 \mathrm{kHz}$ input frequency for (a) and (b), $124 \mathrm{kHz}$ for (c) and (d) and $166 \mathrm{kHz}$ for (e) and (f) 
Acknowledgements The authors would like to thank Eldorado Research Institute for supporting this work.

Data Availability Statement Data sharing not applicable to this article as no datasets were generated or analysed during the current study.

\section{Declaration}

Conflict of interest Partial financial support was received from Eldorado Research Institute.

Open Access This article is licensed under a Creative Commons Attribution 4.0 International License, which permits use, sharing, adaptation, distribution and reproduction in any medium or format, as long as you give appropriate credit to the original author(s) and the source, provide a link to the Creative Commons licence, and indicate if changes were made. The images or other third party material in this article are included in the article's Creative Commons licence, unless indicated otherwise in a credit line to the material. If material is not included in the article's Creative Commons licence and your intended use is not permitted by statutory regulation or exceeds the permitted use, you will need to obtain permission directly from the copyright holder. To view a copy of this licence, visit http://creativecommons. org/licenses/by/4.0/.

\section{References}

1. Razavi, B. (2020). Lower bounds on power consumption of clock generators for ADCs. ISCAS. https://doi.org/10.1109/ ISCAS45731.2020.9180391

2. Redmayne, D., Trelewicz, E., \& Smith, A. (2006). Understanding the Effect of Clock Jitter on High Speed ADCs. Linear Technologyhttps://www.analog.com/media/en/reference-design-doc umentation/design-notes/dn1013f.pdf.

3. Razavi, B. (2020). Design of CMOS Phase Locked Loops From Circuit Level to Architecture Level. Cambridge: Cambridge University Press.

4. Hanumolu, P., Brownlee, M., Mayaram, K., \& Moon, U. K. (2004). Analysis of charge-pump phase-locked loops. IEEE Transactions on Circuits and Systems I: Regular Papers, 51(9), 1665-1674. https://doi.org/10.1109/TCSI.2004.834516

5. de la Rosa, J. M. (2018). CMOS Sigma-Delta Converters Practical Design Guide (2nd ed.). Wiley.

6. Sai, A., et al. (2008). A low-jitter clock generator based on ring oscillator with $1 / \mathrm{f}$ noise reduction technique for next-generation mobile wireless terminals. A-SSCC, 425-428.

7. Shi, X., et al. (2006). A low-jitter and low-power CMOS PLL for clock multiplication. ESSCIRC, 174-177.

8. Sahu, H., Paliwal, P., Yadav, V., \& Gupta, S. (2016). A low-jitter digital-to-time converter with look-ahead multi-phase DDS. (pp. 219-222). https://doi.org/10.1109/LASCAS.2016.7451049

9. Volobuev, P. S., Fedorov, R. A., Poryadina, M. V., Ryzhova, D. I., \& Gavrilov, S. (2019). A low-jitter 300mhz cmos pll for double data rate applications. (pp. 1631-1635). https://doi.org/10. 1109/EIConRus.2019.8656683

10. Bettini, L., Christen, T., Burger, T., \& Huang, Q. (2015). A reconfigurable dt $\delta$ modulator for multi-standard $2 \mathrm{~g} / 3 \mathrm{~g} / 4 \mathrm{~g}$ wireless receivers. IEEE Journal on Emerging and Selected Topics in Circuits and Systems, 5(4), 525-536. https://doi.org/10.1109/ JETCAS.2015.2502162

11. Morgado, A., \& del Río, R., de la Rosa, J.M., Bos, L., Ryckaert, J., Van der Plas, G.: A, . (2010). $100 \mathrm{khz}-10 \mathrm{mhz}$ bw (78th-to- 52db dr, ed., pp. 418-421). https://doi.org/10.1109/ESSCIRC. 2010.5619732

12. Wu, J., et al. (2009). A low-jitter distributed synchronous clock using DAC. IEEE-NPSS Real Time Conference

13. Chen, Y., et al. (2013). Low jitter clock driver for high-performance pipeline ADC. ASICON

Publisher's Note Springer Nature remains neutral with regard to jurisdictional claims in published maps and institutional affiliations.

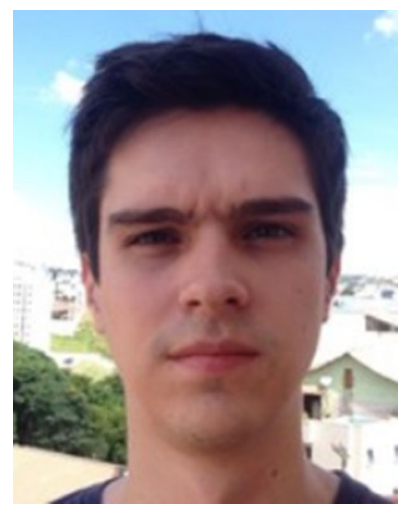

Mateus Castro Received his Bachelor's Degree in Electrical Engineering from Federal University of Juiz de Fora (UFJF) and has certification in analog/mixed-signals (AMS) IC design from the National Program of Integrated Circuits Designers (IC-Brazil Program). Currently he is a M.Sc. student in microelectronics at University of Campinas (Unicamp).

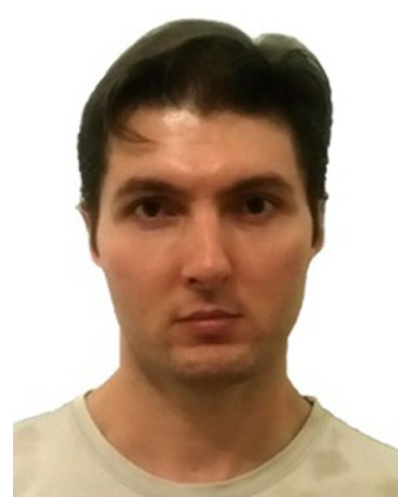

Raphael Souza Received his Bachelor's degree in Electrical Engineering from Federal University of Rio Grande do Sul (UFRGS), his Master's degree in power electronics from Pontifical Catholic University of Rio Grande do Sul (PUCRS), has certification in Analog and Mixed-Signal (AMS) and Radio Frequency (RF) integrated circuits (IC) design from the National Program of Integrated Circuits Designers (IC-Brazil Program), and his Ph.D. degree in microelectronics from University of Campinas (Unicamp).

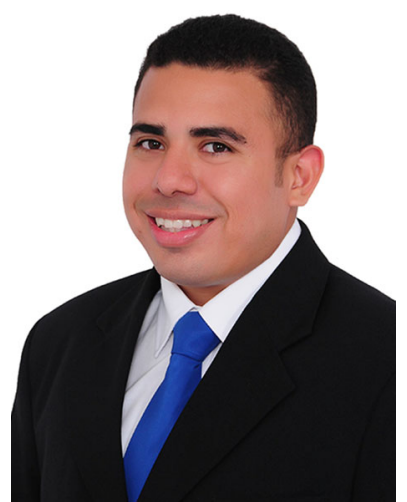

Agord Junior Received the graduate in computer engineering at Potiguar University (UnP). Has certification in radio frequency $(\mathrm{RF})$ integrated circuits design from IC-Brazil Program. He received the M.S. in electrical engineering from the University of Campinas (Unicamp). He is a Ph.D. student with the Department of Electrical Unicamp. 


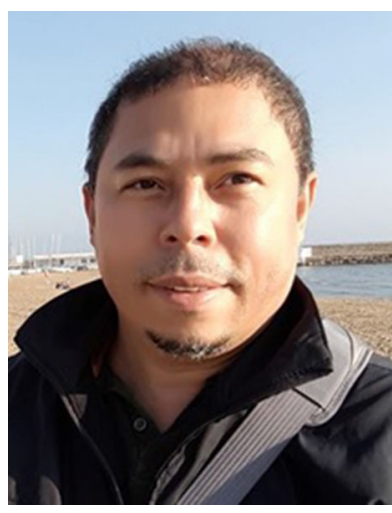

Eduardo Lima Received the DEA and Ph.D. degree in Telecommunications from the Universitat Politècnica de València, UPV, Spain, in 2006 and 2016, respectively. $\mathrm{He}$ worked at Ericsson of Brazil, 2000-2002, LINCS-Brazil, 2007-2008, and Idea! Electronics Systems, 2009-2011, with Telecommunications, FPGAs, and ASICs. He is Collaborator Professor at Unicamp and is currently working at Eldorado Research Institute, where he is the manager of the Hardware Design department. His current research interests include integrated transceiver circuits for wireless, wireless networks, embedded systems, signal processing, sensing, AI and FPGA applications.

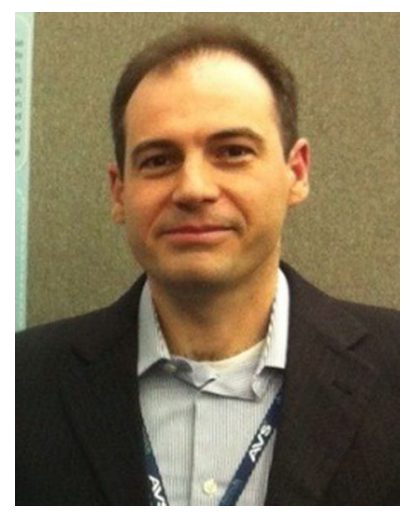

Leandro Manera Received his Bachelor's degree in Electrical Engineering from Lins Engineering School (EEL) at Lins$\mathrm{SP}, \mathrm{Ph} . \mathrm{D}$. Masters and Electronic Engineer in electrical engineering from University of Campinas (Unicamp), in Campinas-SP, Brazil. Currently he is a Professor in electrical and computer engineering in Semiconductors, Instruments and Photonics Department at School of Electrical and Computer Engineering (DSIF/FEEC/ Unicamp). He is also responsible for the Laboratory of Electronic and RF Solutions (LSERF) at FEEC. His research interests include RF measurements, semiconductors devices and antenna design. 